vol. $20-n^{\circ} 1 \mid 2004$

Numéro ouvert

\title{
LORCERIE Françoise, L'école et le défi ethnique. Éducation et intégration
}

\section{Christian Poiret}

\section{(2) OpenEdition}

1 Journals

Édition électronique

URL : https://journals.openedition.org/remi/4033

DOI : $10.4000 /$ remi.4033

ISSN : $1777-5418$

Éditeur

Université de Poitiers

\section{Édition imprimée}

Date de publication : 1 mai 2004

Pagination : 209-210

ISBN : 2-911627-36-9

ISSN : 0765-0752

Référence électronique

Christian Poiret, « LORCERIE Françoise, L'école et le défi ethnique. Éducation et intégration », Revue européenne des migrations internationales [En ligne], vol. 20 - $n^{\circ} 1$ | 2004, mis en ligne le 24 septembre 2008, consulté le 16 avril 2022. URL : http://journals.openedition.org/remi/4033 ; DOI : https://doi.org/ 10.4000/remi.4033

Ce document a été généré automatiquement le 16 avril 2022.

(C) Université de Poitiers 


\title{
LORCERIE Françoise, L'école et le défi ethnique. Éducation et intégration
}

\author{
Christian Poiret
}

\section{RÉFÉRENCE}

LORCERIE Françoise, L'école et le défi ethnique. Éducation et intégration. Paris, 2003, INRPESF, collection Actions Sociales/Confrontations. ISBN : 2710115921

1 Avec L'école et le défi ethnique, Françoise Lorcerie comble un vrai manque en nous fournissant une analyse de l'institution scolaire française du point de vue des relations interethniques - et non pas des relations interculturelles, car comme le souligne l'auteur, le concept d'ethnicité ne parle pas de culture mais de statut - ainsi qu'un tour d'horizon de la production des sciences humaines et sociales en la matière. Car un des points forts de cet ouvrage tient à son approche résolument pluridisciplinaire, tant dans la présentation synthétique d'un paradigme de l'ethnicité que dans les contributions des différents auteurs qui sont mobilisés pour présenter et analyser ses manifestations dans l'espace scolaire.

Dans une première partie, Françoise Lorcerie expose les bases épistémologiques et conceptuelles d'une théorie de l'ethnicité, en s'appuyant sur les acquis de la sociologie, de la psychologie sociale, de l'histoire et de la philosophie politique. Elle dégage ainsi un "paradigme psycho-socio-politique", articulant quatre noyaux théoriques: le premier, relatif à l'identité sociale, présente des outils d'analyse des dynamiques d'appartenance catégorielle; le deuxième, en recourant aux théories de la domination symbolique et du stigmate, replace les processus de catégorisation ethnique dans les dynamiques de classement liés à la domination sociale; le troisième porte sur l'évolution historique des distinctions ethniques, en s'appuyant sur une analyse critique des deux grands schèmes explicatifs, les théories assimilationistes (Glazer, Moynihan, Parsons) et les théories non-évolutionistes de l'assimilation (Weber, Barth); 
le quatrième et dernier noyau, interroge la dimension politique de l'ethnicité et en particulier sa place dans l'unité nationale et son rapport à l'État-nation.

3 Les cent premières pages du livre constituent donc un état des lieux très clair avec de multiples références tant parmi les "classiques» qu'à partir de recherches plus récentes. On appréciera en particulier, la capacité de l'auteure a présenter pédagogiquement, les débats contemporains de la philosophie politique qui opposent "communautariens » et "libéraux » quant aux nouvelles normes démocratiques permettant de prendre en compte le caractère fondamentalement pluriel des sociétés contemporaines.

4 La deuxième partie (pp. 100-159) est constituée d'un ensemble d'articles dans lesquels différents auteurs fournissent des données empiriques qui dépeignent certains des principaux « faits scolaires » relevant du paradigme de l'ethnicité.

5 La troisième partie (pp. 161-227) revient sur l'application du paradigme de l'ethnicité à l'univers scolaire en regroupant les contributions de chercheurs de diverses disciplines qui abordent certaines des grandes questions que celle-ci soulève : l'articulation de la problématique sociale de l'ethnicité avec la psychologie clinique d'origine freudienne ; l'identification ethnique comme ressource mobilisable par les acteurs dominés; le lien entre l'ethnicisation de l'espace scolaire et les politiques d'intégration scolaires des enfants de migrants. Cette partie s'ouvre par un chapitre dans lequel Françoise Lorcerie fait une synthèse de ce cadre théorique appliqué à l'école, en articulant trois niveaux d'analyse qui interagissent entre eux : celui des interactions entre le statut ethnique des individus et leur rôle social ; celui de l'influence des pratiques scolaires sur le statut ethnique des individus ou sur la mise en saillance de l'ethnicité dans les relations scolaires; celui de la relation entre l'ethno-nationalisme et la domination d'une part et les structures, les formes et les contenus d'éducation d'autre part. Cette synthèse est prolongée et illustrée par un fort utile bilan thématique des recherches en cours dans ce domaine.

6 La quatrième et dernière partie (pp. 230-309), placée sous le signe de la philosophie politique, revient sur la crise de l'identité nationale dans les sociétés pluriethniques et sur les réponses éducatives qui peuvent être apportées aux discriminations et aux affirmations identitaires. Les trois premiers chapitres présentent cette problématique ( «a philosophie politique face aux tensions ethniques », «L'école et les mémoires de l'immigration", "Revisiter l'“éducation interculturelle" "), tandis que les quatre suivants décrivent et analysent des pratiques pédagogiques très intéressantes qui sont autant de tentatives de réponse à l'ethnicisation des relations scolaires. Enfin, le livre s'achève par une bibliographie thématique très complète (pp. 313-333) qui n'est pas le moindre des atouts de ce travail.

7 Au total, L'école et le défi ethnique est un ouvrage agréable à lire, qui propose à la fois une analyse synthétique des théories des relations interethniques - qui en fait un outil précieux et susceptible d'intéresser un public qui ne se limite pas aux spécialistes de l'école - et une présentation des recherches et des pratiques concernant l'ethnicité dans l'institution scolaire, très utile pour les enseignants comme pour les étudiants et les chercheurs spécialisés dans ce domaine.

8 Raisons de plus pour regretter la brièveté des paragraphes consacrés à la race et aux processus de racisation (p. 37-38), aux dispositifs français de lutte contre les discriminations (p. 96) ainsi qu'à l'apport de la statistique publique à la connaissance des discriminations - non seulement le débat n'est pas clos quant à l'introduction dans 
les grandes enquêtes publiques d'une "variable ethnique » (qui ne saurait d'ailleurs se confondre à la variable "immigré " construite par l'INED), mais, de surcroît, l'introduction de celle-ci ne saurait, à elle seule, suffire à apporter la preuve du caractère ethniste ou raciste des discriminations constatées, comme le montre l'analyse de la situation britanique ${ }^{1}$. Plus généralement, le cadre théorique aurait été encore enrichi par un approfondissement des difficultés d'articulation entre macro-analyse et micro-analyse. Il reste donc à espérer que le succès du livre appelle une réédition qui permettra à l'auteure de présenter les approches constructivistes du passage de l'individuel (catégorisation et dynamiques identitaires) au collectif (rapports de domination, institutionnalisation d'un ordre social inégalitaire) et les possibilités offertes par le recours à certains concepts opératoires comme celui de discrimination institutionnelle que ne compense pas vraiment l'utilisation d'un schéma fléché (p. 164).

\section{NOTES}

1. Cf. par exemple, Wrench, J. 1997, «Des problèmes de passage de l'école à l'emploi chez les jeunes issus de l'immigration au Royaume Uni » in Aubert, Tripier, Vourc'h (dir) Jeunes issus de l'immigration : de l'école à l'emploi, Paris, Ciemi-l'Harmattan : 131-140.

\section{AUTEURS}

CHRISTIAN POIRET

Urmis-Paris 